according to figure 2 , if weight gain is roo $\mathrm{g}$ higher than average and liveweight $250 \mathrm{~g}$ higher than evarage, feed conversion remains unchanged/table $2 /$. Therefore genetical improvement of feed conversion remains unchanged/table $2 /$. Therefore genetical improvement of feed conversion by indirect selection is, in the author's judgement, better than separate selection for the two properties.

\title{
LE PROGRAMME MEXICAIN DE SELECTION DU IAPIN : OBJECTIFS ET PREMIERS RÉSULTATS
}

\author{
A. PAEZ CAMPOS(*), H. DE ROCHAMBEAU(**), R. ROUVIER(***) \\ et B. POUJARDIEU $(* * *)$
}

(*) Direccion general de avicultura y especies menores, Durango 138 Mexico 6 DF. Mexique

(**) Chaire de Zootechnie, Institut national agronomique Paris-Grignon 16, rue Claude Bernard, 75231 Paris, France

(***) Station d'Amélioration génétique des animaux I.N.R.A., Toulouse, B.P. I2, 31320 Castanet-Tolosan, France

Il s'agit d'un programme de sélection conçu dans le cadre de la coopération scientifique et technique entre la Direccion general de Avicultura $Y$ Especies Menores (DGAFM/Mexique) et la Station d'Amélioration Génétique des animaux (Institut National de la Recherche Agronomique, Toulouse, France). L'objectif est de constituer à partir du matériel animal local, des lignées nationales pour la production de viande et de fourrure utilisables en élevages rationnels ou fermiers. Les animaux seront sélectionnés dans le Centre National de Cuniculture d'Irapuato (État de Cuanajato). Ils sont identifiés au sevrage puis tatonés lors de leur mise à la reproduction. I eurs performances sont collectées sur un système de 3 fiches : "fiche femelle ", "fiche mâle ", " fiche portée ". Nous avons évalué la productivité numérique jusqu'au sevrage đes 4 souches présentes à Irapuato. Nous avons constitué deux lignées femelles (Néo-Zélandais Blanc et Chinchilla) sélectionnées sur le nombre de lapereaux sevrés par mois de production. La sélection se fait chaque semaine lors du sevrage. Nous travaillons donc en générations chevauchantes. Chaque lignée est divisée en 16 groupes de reproductioni de 16 femelles et 2 mâles.

Ces groupes sont soumis à des règles d'accouplement et de renouvellement bien précises. Nous avons aussi constitué plusieurs lignées mâles d'après le gain moyen quotidien entre le sevrage et $7 \mathbf{I}$ jours. La lignée Rex a été réduite à 3 génotypes de coloration : Castor, Chinchilla et Beige. Nous allons maintenant tester les divers croisements entre ces lignées dans plusieurs environements pour vérifier qu'elles sont ađaptées aux conditions des diverses régions du Mexique.

ÉTUDE D'UNE UNITÉ DE GRANDS-PARENTAUX DANS UN ÉLEVAGE, DE LAPINS HYBRIDES, PREMIERS RÉSULTATS

M. COLIN, H. ROULLERE, J. SIMONNET et Y. LUCAS

Société Sanders, I7, quai de l'Industrie, 9T2oo Athis-Mons (France)

L'introduction de lapines grands-parentales dans un élevage de lapins hybrides est l'une des solutions préconisées pour faciliter le renouvellement des reproducteurs.

Pour juger de son intérêt réel, il est nécessaire de connaître les caractéristiques de leur production en comparaison de celles de lapines parentales.

Pour cela, nous avons étudié pour un croisement commercial (Hyla) trois traitements :

- des lapines gratids-parentales sailles ro jours après 1a mise-bas (un essai préliminaire nous ayant montré de nombreux inconvénients à faire saillir ces lapines post-partum);

- des lapines parentales également saillies ıo jours après la mise-tas;

- des lapines parentales saillies post-partum. 

nients :

Nous avons ainsi montré que les lapines grands parentales présentent 2 types d'inconvé-

- une baisse de prolificité et une augmentation de la mortalité des lapereaux avant sevrage, aboutissant à une diminution de l'effectif des lapereaux sevrés par portée;

- l'obligation d'adopter pour ces femelles grands-parentales la saillie ro jours après la mise-bas. Cette impossibilité de saillir les lapines post-partum diminue fortement le tombre de misesbas par cage mère et par an.

Il en résulte globalement une forte diminution de la production annuelle de lapereaux dans les cages occupées par les lapines grands-parentales par rapport aux cages occupées par les lapines parentales.

Dans le croisement Hyla, cette production par cage femelle est ainsi évalué à 52,6 lapereaux sevrés par an pour les lapines grands-parentales quand elle est de 59,6 pour les lapines parentales saillies ro jours après la mise-bas et 64,5 pour les parentales saillies post-partum.

\title{
ESTUDIO BIOMETRICO DE I,A FERTIIIDAD DE LAS CONEJAS FN I,A RIBERA DEL EBRO DE I.A PROVINCIA DE LOGROÑO
}

\section{J. CUEVAS ALVAREZ}

\author{
Jefatura de Producción Animal \\ C/Vázquez de Mella, 8, $7^{\circ}$ - Zaragoza (España)
}

Se ha realizado el estudio del Indice de Fertilidad de la Coneja Reproductora de una explotación de la Ribera del río Eibro en la provincia de Logroño.

El estudio ha tenido lugar sobre los resultados obtenidos en 2255 cubriciones, y se han determinado los datos biométricos de dicha población.

Hallándose la media o promedio, el error de la media, la desviación tipo y el cœficiente de variación y referidos a los distintos meses del año.

Ell I.F. medio obtenido a lo largo del año, en explotaciones semi-industriales, en ambiante variable, y en hembras no seleccionadas, se estima en el 61 p. 100.

I as cubriciones con Indice de Fertilidad más alto son las realizadas en los meses de marzo, abril, mayo y junio. Las cubriciones con Indice de Fertilidad más bajo son las realizadas en los meses de septiembre, octubre, noviembre y diciembre referidas a la Zona de la Ribera del Ebro en la provincia de Iogroño (España).

\section{COMPARAISON DE IA REPRODUCTION DE LAPINS DE DEUX GÉNOTYPES EFFETS DE L'AGE ET DE LA SAISON}

Françoise HULOT et G. MATHERON

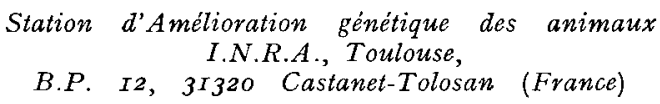

Une comparaison de deux génotypes de lapines Californien et Néo-Zélandais Blanc, sur les composantes de la taille de portée conduit aux conclusions suivantes : $(i)$ Une femelle Californienne quel que soit son âge et la saison de mesure pond en moyenne deux ovules de plus qu'une Néo-Zélandaise blanche. (ii) L'étude de la viabilité préimplantatoire donne un classement inverse de ces deux races. (iii) Lorsque l'âge d'une femelle augmente, son niveau ovulatoire augmente et sa capacité utérine diminue. (iiii) C'est au stade primipare qu'il y a le plus d'embryons suite à une conjonction favorable des deux mécanismes antagonistes. (iiiii) L'effet de la saison the donne pas des résultats homogènes selon le caractère considéré. 